\section{$\underset{\substack{\text { hommes } \\ \text { \& migrations }}}{ }$}

\section{Hommes \& migrations}

Revue française de référence sur les dynamiques

migratoires

$1319 \mid 2017$

Réfugiés et migrants au Liban

\title{
Les services de santé mentale pour les réfugiés syriens
}

Entre politiques de l'humanitaire et politiques du soin

\section{Filippo Maria Marranconi et Hala Kerbage Hariri}

\section{(2) OpenEdition}

Journals

Édition électronique

URL : http://journals.openedition.org/hommesmigrations/3977

DOI : $10.4000 /$ hommesmigrations.3977

ISSN : 2262-3353

Éditeur

Musée national de l'histoire de l'immigration

Édition imprimée

Date de publication : 1 octobre 2017

Pagination : 87-94

ISBN : 978-2-919040-39-1

ISSN : 1142-852X

Référence électronique

Filippo Maria Marranconi et Hala Kerbage Hariri, «Les services de santé mentale pour les réfugiés syriens », Hommes \& migrations [En ligne], 1319 | 2017, mis en ligne le 01 octobre 2020, consulté le 08 janvier 2021. URL : http://journals.openedition.org/hommesmigrations/3977 ; DOI : https://doi.org/ ERREUR PDO dans /localdata/www-bin/Core/Core/Db/Db.class.php L.34 : SQLSTATE[HYO00] [2006] MySQL server has gone away 


\section{LES SERVICES DE SANTÉ MENTALE POUR LES RÉFUGIÉS SYRIENS : \\ ENTRE POLITIQUES DE L'HUMANITAIRE ET POLITIQUES DU SOIN}

Par FILIPPO MARIA MARRANCONI, anthropologue, doctorant en anthropologie, EHESS (Paris), IIAC/LAUM, doctorant associé à l'IFPO (Institut français du Proche-Orient) et HALA KERBAGE Hariri, psychiatre, chargée de cours, département de psychiatrie, Faculté de médecine, université Saint-Joseph de Beyrouth.

L'épreuve anxiogène de l'exil, l'expérience de I'habitat informel, une survie qui dépend des organisations non gouvernementales : voici un contexte psychologique explosif auquel sont soumis les réfugiés syriens au Liban. Cette étude consacrée au point de vue des professionnels de santé libanais révèle la construction d'une image particulière de leurs patients syriens : celle de victimes ayant besoin d'aide. Loin de permettre de traiter les pathologies mentales provoquées par cette situation délétère, cette attitude du corps médical à l'égard des patients syriens tend paradoxalement à nourrir une vision négative de soi.

Dans le contexte de la crise syrienne au Liban, de multiples organisations non gouvernementales (ONG) locales et internationales, en coordination avec le ministère de la Santé publique et le ministère des Affaires sociales, proposent aux réfugiés syriens des services de santé mentale et de support psychosocial (Mental Health and Psychosocial support services, MHPSS), d'après les recommandations de l'InterAgency Standing Committee ${ }^{1}$ (IASC). En effet, l'expérience de la souffrance dans la population des réfugiés au Liban reste aujourd'hui principalement l'apanage d'une gestion médicale et humanitaire et, dans ce contexte, les « disciplines psy » occupent une place d'envergure dans la définition de cette expérience. Peu de travaux abordent ce sujet dans sa dimension sociale ${ }^{2}$ au delà du regard médical et il n'existe pas de données sur l'expérience des réfugiés syriens avec les services de santé mentale ou, de façon plus générale, sur les idiomes de la souffrance mobilisés par cette population. Une revue de littérature commanditée par le Haut Commissariat des Nations unies pour les 
réfugiés (HCR) tente d'informer sur des spécificités socio-culturelles propres au contexte syrien, afin de pouvoir adapter les services ${ }^{3}$, mais il n'y a pas, à notre connaissance, d'études ayant impliqué des réfugiés syriens eux-mêmes dans le but d'explorer leur expérience au-delà des catégories diagnostiques. Les études qui existent sont surtout des études épidémiologiques recherchant les prévalences de diagnostics psychiatriques dans cette population, notamment de syndrome de stress post-traumatique (PTSD) et de dépression ${ }^{4}$; les moyens de diagnostic utilisés étant des échelles standardisées non validées dans le contexte syrien (comme, par exemple, le Mini international neuropsychiatric interview-MINI) qui sont basées sur une liste de symptômes.

En se concentrant sur les symptômes plutôt que sur l'expérience, sur les pourcentages de diagnostics plutôt que sur le vécu individuel, ces études réduisent le vécu des réfugiés à une liste de symptômes isolés de leur contexte, qu'il suffit de quantifier et de mesurer pour diagnostiquer un trouble psychiatrique. Il en résulte une surestimation de la fréquence de PTSD et de dépression, occultant ainsi le fait que ces manifestations peuvent être une réaction normale à des circonstances de vie extrêmes et réalisant une médicalisation de la souffrance.

Cependant, des critiques ont progressivement émergé vis-à-vis de ces approches, qui risquent d'enfermer les réfugiés dans un rôle passif tout en pathologisant une souffrance ordinaire et en éradiquant tout contexte social et historique dans lequel cette souffrance s'inscrit ${ }^{5}$.

\section{Une étude sur la construction médicale et psychologique du réfugié}

Vu les limites des études précédemment citées, ainsi que fort de notre propre expérience clinique auprès de réfugiés syriens et de notre recherche dans le milieu psychiatrique, nous avons entrepris en 2016 une étude dont le but a été d'explorer les politiques de mise en place des services de santé mentale, à travers une analyse de la documentation disponible, et du discours que les psychiatres et les autres professionnels de santé mentale construisent autour de la population réfugiée. Au centre de notre intérêt se trouve l'expérience que les réfugiés ont de ces services, ainsi que les manières dont l'expérience de la souffrance prend forme dans leur contexte de vie. Les données dont on fait usage dans cet article ont été recueillies au cours d'une enquête de terrain menée durant vingt quatre mois (2014-2015) dans le cadre de la recherche doctorale de l'un des deux auteurs, ainsi que pendant huit mois dans l'année 2016 dans le cadre de notre étude. Nous avons contacté les dix ONGs fournissant des services MHPSS spécialisés pour adultes (consultations de psychiatrie et/ou de psychothérapie). Seules deux ONG (internationales) nous ont permis l'accès aux réfugiés syriens bénéficiant de leurs services, basés dans les régions de la Bekaa et de Beyrouth, qui concentrent le plus grand nombre de réfugiés syriens au Liban ${ }^{6}:$ Médecins du Monde ${ }^{7}$, fournissant des services à La Bekaa, et une autre

3. United Nations High Commissioner for Refugees (UNHCR), Culture, context and the mental health and psychosocial wellbeing of Syrians, Geneva, 2015. 4. Francois Kazour et al., "Post-traumatic stress disorder in a sample of Syrian refugees in Lebanon ", in Comprehensive Psychiatry, $n^{\circ}$ 72, 2017, pp. 41-47; Souaiby Lama et al., " Impact of the syrian crisis on the hospitalization of Syrians in a psychiatric setting ", in Community Mental Health Journal, vol. 52, $n^{\circ}$ 1, 2016, pp. 84-93; Wadih Naja et al., "Prevalence of dépression in Syrian refugees and the influence of religiosity", in Comprensive Psychiatry, n 68, 2016, pp. 78-85; E. G. Karam et al. " Lebanon : mental health system reform and the Syrian crisis ", in British Journal of Psychiatry, vol. 13, $n^{\circ}$ 4, 2016, pp. 87-89. 5. Lisa H. Malkki, "Speechless Emissaries : refugees, humanitarianism and dehistoricization ", in Cultural Anthropology, vol. 11, $n^{\circ}$ 3, 1996, pp. 377-404; Dereck Summerfield, "A critique of seven assumptions behind psychological trauma programmes in war-affected areas ", in Social Science \& Medicine, vol. 48, n 10, 1999, pp. 1449-1462; Didier Fassin, Richard Rechtman, L'empire du traumatisame: enquete sur la condition de victime, Paris, Flammarion, 2011 ; Roberto Beneduce, Etnopsichiatria. Sofferenza mentale e alterità fra Storia, dominio e cultura, Roma, Carocci, 2007 ; Archeologie del Trauma : Un'Antropologia del Sottosuolo, Roma-Bari, Laterza Edizioni, 2010. 6. United Nations High Commissioner for Refugees (UNHCR), Syria Regional Refugee Response, 2017. 7. Nous remercions Médecins du Monde qui nous a permis l'accès aux Syriens bénéficiant de ses services, ainsi que tous les participants qui ont accepté de nous recevoir pour des entretiens. Cette recherche a été conduite de manière indépendante par les deux auteurs. Cet article reflète leur point de vue et non pas celui de Médecins du Monde. 
ONG ayant souhaité garder l'anonymat. Les huit autres ONGs ont invoqué des politiques strictes de confidentialité ainsi que des craintes d'exposer leurs bénéficiaires "vulnérables " aux risques "d'entretiens avec des inconnus qu'ils ne reverront plus ${ }^{8}$ ». Cependant, toutes les ONGs concernées ${ }^{9}$ ont accepté que nous fassions des entretiens avec le personnel soignant et/ou les coordinateurs des programmes, sous couvert d'anonymat ${ }^{10}$.

Dans cet article, qui constitue une première réflexion sur le sujet abordé, nous analysons les discours mobilisés par certains professionnels de la santé mentale afin de montrer la construction du réfugié syrien dans un contexte de gestion humanitaire. L'analyse de ces discours - récurrents dans notre ethnographie bien qu'ils ne soient pas partagés par tous les professionnels - nous permet d'explorer le fonctionnement des relations entre soignants et soignés, et de révéler certains malentendus, difficultés et angles morts qui sillonnent la pratique thérapeutique. Enfin, à travers une remise en contexte de la rencontre thérapeutique, nous montrons comment les discours et les stéréotypes mobilisés par les professionnels, assignant une place particulière au sujet et à son expérience de la souffrance, dessinent une véritable politique du $\operatorname{soin}^{11}$.

\section{Le Syrien dérangeant: la consanguinité ou l'ignorance de la culture}

«... Ils ont une mauvaise odeur... Tous les réfugiés
syriens ont une mauvaise odeur. Surtout parce que les femmes sont habillées beaucoup, et il y a l'odeur de la sueur... L'aversion qu'on ressent pour eux, ce r'est pas à cause de la guerre, tant qu'ils n'ont pas participé aux combats [de la guerre civile]... Mais les femmes, c'est surtout l'image de l'ignorance, et c'est une image très ancrée. Les Syriens sont ignorants. Et il y a l'histoire de loccupation du Liban par la Syrie : si cétait les Français ou les Américains, on veut bien, parce qu'ils sont plus civilisés... mais là, il y a un contre-transfert négatif, ce sont des gens qui ne méritent pas le respect ${ }^{12} \ldots$ »

Dans cette déclaration, on entrevoit certains des traits qui forment l'image dévalorisante du réfugié Syrien au Liban et de ce que Vincent Geisser, en parlant des relations entre les deux pays, a appelé «réveil des fantômes du passé $e^{13}$ ». À partir du terme "réfugié », qui évoque la présence palestinienne, la guerre civile libanaise, l'image du travailleur immigré et l'occupation syrienne du pays jusqu'en 2005, la figure du syrien réveille dans la classe politique, comme dans la population libanaise, des attitudes et des sentiments ambivalents, qui nécessitent d'être analysés historiquement et dans leur actualité politique. Mais la relation entre professionnels et usagers est traversée aussi par d'autres stéréotypes qui se mélangent et à la fois dépassent celui du syrien comme réfugié indésirable, pour faire de lui un sujet essentiellement dénué et contraint par la pauvreté, une figure rurale en proie à l'ignorance et au traditionalisme, incapable d'exprimer ses besoins et nécessitant de l'éducation.

Certains des professionnels interviewés se plaignent de l'ignorance des Syriens, de leur faible taux d'alphabétisation ou bien de leur incapacité à lire en lettres latines, et donc de la nécessité continue d'avoir à leur expliquer le nom et la fonction 
des médicaments, en passant par les procédés à suivre pour leur prise : «Leur culture les empêche de comprendre que prendre le médicament une fois ne suffit pas, qu'il faut attendre pour guérir ${ }^{14}$. " L'ignorance des réfugiés apparaît comme un facteur déterminant, également perçu comme étant la raison du haut taux de natalité dans cette population, ce qui met indirectement en cause la capacité d'accomplissement du rôle parental des réfugiés. Dans des conditions de pauvreté extrême, les Syriens continuent d'enfanter, ce qui est interprété de la part de certains professionnels comme une " manière irrationnelle et illogique ${ }^{15}$ » d'affronter les conditions de vie puisqu'ils n'ont pas les moyens financiers de s'occuper de leurs enfants. Bien que certains professionnels reconnaissent les difficultés économiques qui empêchent les parents de payer la scolarité de leurs enfants, une partie d'entre

L'ignorance des réfugiés apparaît comme un facteur

déterminant, également perçu comme étant la raison du haut taux de natalité dans cette population, ce qui met indirectement en cause la capacité d'accomplissement du rôle parental des réfugiés. eux trouve la source de ce comportement dans le désintérêt que les Syriens auraient pour l'éducation.

L'ignorance, selon certains, joue aussi un rôle, du moins indirect, dans les explications génétiques concernant la fréquence de certaines pathologies (schizophrénie, trouble bipolaire, retard mental, autisme) dans la population des réfugiés. En effet, selon ces professionnels, le taux de consanguinité serait très élevé dans la population syrienne. Bien qu'il ne soit pas évoqué comme l'unique facteur à prendre en compte pour expliquer les situations pathologiques, il constituerait néanmoins une condition de possibilité fondamentale pour appréhender les difficultés des réfugiés et, notamment, les échecs scolaires de leurs enfants. Selon une psychiatre interrogée :

«- Les Palestiniens sont une population très particulière: ils ont un taux de consanguinité très élevé. Plus que les Libanais. Le Liban est le pays avec le taux de consanguinité le plus bas du monde arabe. (...) Quand tu te maries avec un cousin germain, tu as plus de risque d'avoir des maladies psychiatriques, plus de schizophrénies, plus de troubles bipolaires. En plus ils sont très défavorisés, le système éducatif est nul. Il y a un taux de retard mental très élevé. (...) Moi, je vois des Syriens aussi, mais je vois plus des Palestiniens de Syrie (...). Ils se marient encore plus entre eux, et il y a un taux d'analphabétisme très élevé.

- Tu veux dire qu'ils sont stupides?

- Il y a un retard chez eux. Pas tous, mais la majorité. Je ne dis pas qu'ils sont retardés, mais ils n'arrivent pas à passer le brevet. (...) Tu sais, tes acquisitions scolaires vont avoir une répercussion sur ton niveau intellectuel. Ce que tu apprends va stimuler tes neurones, t'ouvrir des horizons. Eux, ils quittent l'école tôt et ils vont faire des petits boulots. (...) Moi, je vois plein d'enfants de 6-7 ans qui ne vont pas à l'école. Ils essayent, mais ils n'arrivent pas à intégrer le système scolaire, ils sont découragés et ils quittent ${ }^{16}$.

Finalement, la consanguinité est un concept utilisé de manière très ambiguë. Si certains psychiatres lui reconnaissent un impact direct sur la santé, d'autres la considèrent comme la conséquence d'une culture fondamentalement traditionnelle et arriérée. Néanmoins, dans les deux cas, suivant ce raisonnement, il est donc possible d'établir une hiérarchie des populations (libanais, syriens, palestiniens, palestiniens de Syrie...) sur la base de leur supposé degré de consanguinité. Cette hiérarchie différentielle, subrepticement évolutionniste et ethnicisante, qui recoupe, au niveau génétique, le degré de désavantage de chaque groupe et leur degré de développement ou d'éducation, est en réalité basée sur le découpage des statuts. Une hiérarchie qui, de manière plus ou moins explicite, établirait un continuum ayant à ses pôles la tradition et la modernité.

\section{Professionnels universels, Syriens particuliers}

Le rôle de la culture dans le discours des psychiatres est généralement évoqué de deux manières. Premièrement, le modèle de la culture comme épiphénomène, comme une manière alternative 
d'exprimer le malaise. Afin de découvrir la vraie pathologie sous-jacente, il faudrait sélectionner les symptômes cohérents avec le cadre de lecture psychiatrique, en incorporant ou en excluant le langage du patient dans la nosologie. Deuxièmement, elle est évoquée par certains psychiatres comme un obstacle qui empêcherait le patient de comprendre le langage psychiatrique, son importance, et d'en suivre les protocoles. Dans les deux cas, le terme de « culture »-mobilisé comme synonyme de particulier (opposé à universel), tradition - est utilisé en opposition à celui de "science psychiatrique », et prend l'allure de ce que Bateson appelle "boite noire » ou « hypothèse dormitive : Pourquoi l'opium provoque le sommeil ? Parce qu'il contient un principe dormitif ${ }^{17}$. " On est donc face, à une cause réifiée qui explique peu et s'auto renforce. Cette production d'hypothèses dormitives - qu'on retrouvera ailleurs - permet à certains professionnels d'ancrer une différence dans une dimension naturelle, ou, pour ainsi dire, dans une culture naturelle, en faisant fonctionner ainsi la psychiatrie comme une discipline $^{18}$. Le corps du Syrien est «opaque ${ }^{19}$ » : opaque à lui-même, puisque le patient semblerait incapable de comprendre ou d'expliquer ce qui lui arrive dans un langage approprié, et opaque pour les professionnels, puisque son universalité serait cachée par sa culture.

Ceci est bien visible dans la dissociation qui est faite entre le besoin du sujet tels que perçu par les professionnels et ce qu'il exprime. Il l'est également et dans le rôle que les «awareness sessions » ou «sessions de sensibilisation » prennent dans l'organisation des services. En effet, une préoccupation constante de la part des prestataires de services de santé mentale est la nécessité d'éduquer les gens sur le savoir psychologique et psychiatrique. Dans les espaces d'attente des associations ou des centres de soins primaires, on trouve toujours une brochure ou un dépliant expliquant aux usagers les symptômes des maladies psychiatriques, les noms de celles-ci, et les indications sur la conduite à tenir en cas de besoin. À ce propos, une assistante sociale nous parle des « awareness sessions » : "Ils disent : je suis très fatigué, je m'énerve facilement, je ne supporte plus mes enfants... Ils ne disent pas : je suis déprimé. Ils ne savent pas qu'ils sont déprimés, alors que nous on voit les symptômes. (...) Quand on n'atteint pas les chiffres requis (par les bailleurs de fonds), on fait plus de "awareness sessions" pour recruter plus de gens. Les gens ne considèrent pas les services de santé mentale comme une priorité, donc il faut les convaincre ${ }^{20}$. " Pour les Syriens manquant d'éducation ou d'information sur les maladies psychiatriques, les « awareness sessions » ne répondent pas seulement à une nécessité organisationnelle (ou à des impératifs de marché liés aux financements), mais se révèlent être un outil pédagogique visant non seulement une sensibilisation, mais aussi une véritable prise de conscience par le patient de son état et de ses besoins. À travers le manuel du «Mental health Gap ", le dépistage des symptômes, et les "awareness sessions ", les assistantes sociales vont les informer, les sensibiliser et les intégrer en tant que "bénéficiaires » dans les services.

Léducation aux discours des disciplines «psy » va ici de pair avec la question de la résipiscence : l'ignorance de l'état maladif de la part du patient, qui, en psychiatrie, peut être un des symptômes de cet état maladif lui-même, se mêle à l'ignorance «due à la culture », qui empêcherait le Syrien d'être conscient de son état, ou qui le pousserait vers ce que les professionnels évoquent comme une forme de " tabou ou de stigmatisation " de la part des patients à l'égard de la maladie mentale. Il se produit donc ici un court-circuit qui ouvre la possibilité à la pathologisation et à la naturalisation de comportements individuels ou de groupes, considérés sur la base d'instruments de diagnostic, plutôt que par une tentative de compréhension de l'expérience du patient à la lumière aussi de ses conditions de vie. 
Le Syrien, face à certains professionnels, est donc qualifié par des stéréotypes qui le rabattent sur sa situation de pauvreté et dénuement, d'ignorance et de traditionalisme aveugle, liés à une origine (vrai ou prétendue telle) rurale. Face à un Syrien manquant de l'éducation requise, et peu désireux de coopérer pour son bien, la relation soignant-soigné prends les caractéristiques d'une relation paternaliste et moralisatrice, tout comme apparaît légitime le mécontentement du médecin éduqué et bienveillant. Dans ce cadre, la notion de culture, ainsi que la notion de consanguinité, utilisées par les professionnels, permettent d'essentialiser les caractéristiques attribuées au réfugié et d'insérer celui-ci dans une dimension naturelle intemporelle.

\section{Le Syrien mensonger}

"- Les mensonges... Tu peux dire qu'ils sont des menteurs, mais tu ne peux pas commencer une thérapie de cette manière. Une fois, une fille de 21 ans est venue. Elle m'a dit des choses, mais elle mentait, elle ne voulait pas tout me raconter. (...) Elle avait une personnalité spéciale. J'ai essayé de la convaincre de ne pas mentir, et j'ai tout essayé. Après elle est partie. Elle avait une personnalité spéciale qui la faisait mentir.

- Peut être elle cherchait d'obtenir quelque chose ici?

- Oui, d'accord. Mais je suis une psychologue, elle ne peut pas obtenir des biens matériels chez moi ${ }^{21}$. "

En évoquant les frustrations liées à leur métier, les professionnels évoquent souvent les comportements que les patients ont vis-à-vis du travail thérapeutique, notamment la problématique de leur propre manipulation. Les professionnels se plaignent du fait que les réfugiés - conscients de l'importance du savoir médical dans les procédures de réinstallation dans les pays européens ou nord américains - demandent, directement, un rapport médical à présenter au HCR. Ils demandent parfois d'insister dans le rapport sur la gravité de leurs traumatismes. Cette attitude apparaît aussi dans leur demande continue d'aides qui, selon certains professionnels, révélerait une certaine intempérance et dévoilerait le manque d'honnêteté de certains d'entre eux.

"Il y a des gens qui essayent de te manipuler, et ils te provoquent. Ca m'est arrivé une fois, qu'on m'ait dit : "- Si tu nous aides pas, je vais dire à mon mari d'aller combattre avec Daesh. - Madame, je suis une psychologue, je ne peux pas vous aider avec les biens matériels !" Et eux, ils reçoivent déjà les biens, chaque mois, et le loyer. (...) Ils reçoivent tout, mais continuent de demander ${ }^{22}$."

Le comportement du réfugié et le sentiment de manipulation qui en découle semblent mettre en crise le rôle du professionnel lui-même, ainsi que son attitude paternaliste. Cependant, les réponses à cet état sont diverses. Parfois, le comportement des réfugiés est lu à la lumière de catégories moralisantes et, comme on l'a vu, à la lumière d'une conception naturalisante de la culture ou, à l'extrême, comme un état pathologique. D'autres fois, la crise de sens du travail du professionnel semble, par contre, assumée, ouvrant ainsi à des possibilités de compréhension ultérieures.

Le concept d' "économie morale du mensonge ${ }^{23}$ " paraît utile pour décrire ces dynamiques. Il est proposé par Roberto Beneduce, qui l'emploie dans l'étude des stratégies narratives utilisées par les migrants pour faire face à l'arbitraire et à la violence bureaucratique des procédures d'évaluation pour l'obtention de l'asile. Cette notion " propose des sentiers analytiques pour comprendre la signification de comportements ou de narrations qui sont souvent banalisées comme tromperies ou tactiques finalisées à l'obtention d'avantages immédiats ${ }^{24}$ ", et elle peut nous aider à interpréter des pratiques dont la valeur politique est difficile à déterminer. Si, dans notre cas, il ne s'agit pas seulement de procédures que les Syriens doivent affronter afin d'obtenir l'asile, ce concept nous semble utile pour comprendre quelque chose du monde dans lequel les réfugiés 
vivent. Leur quotidienneté est jalonnée par la présence des agences onusiennes et des organisations humanitaires ; il s'agit d'un monde, finalement, sillonné par la tension entre répression et compassion décrite par Didier Fassin ${ }^{25}$.

La décision de faire bénéficier un réfugié des aides matérielles est soumise à la capacité de la part du réfugié de démontrer sa situation de besoin auprès des institutions chargées de ces aides. En effet, le HCR organise véritablement une hiérarchie de la souffrance, hiérarchie à laquelle l'individu (ou le foyer familial) doit montrer son appartenance afin d'avoir accès aux moyens de subsistance. Sans, pourtant, être sûr du résultat. Le certificat médical, l'exposition de blessures, l'affichage d'un état de morbidité, deviennent des modes de légitimation de leurs demandes face aux employés des ONG ou aux autorités compétentes ayant rapport avec la demande d'asile.

Dans nos rencontres, le discours sur le HCR (désigné par "Oumam », "Nations », pour Nations unies, puisqu'il s'agit du UNHCR) est omniprésent. Les Nations unies sont vécues comme une organisation qui peut offrir des avantages, et dont les réfugiés dépendent pour le minimum de survie. Cependant, ses logiques demeurent insondables et sont vécues comme arbitraires et hypocrites. Les Oumam constituent une présence lointaine, et pourtant quotidienne, parfois perçus par les réfugiés syriens comme maître de leur destin puisqu'ils intervenir en leur faveur et les sauver. Mais ils sont aussi des interlocuteurs inaccessibles : «Si cela continue comme ça, je vais me mettre le feu, moi et mes enfants, devant le siège des Oumam à Beyrouth »; "On a faitl'entretien, ils ont dit qu'ils allaient nous rappeler. Entre temps, nos voisins ont été acceptés pour voyager. Mais eux, ils ont une meilleure situation que nous! \es phrases ont souvent été entendues dans la bouche de nos interlocuteurs au cours de notre enquête.

\section{Mensonge contre mensonge}

Dans cette situation de précarité, les Syriens sont constamment forcés de devenir des « créatures mimétiques $^{26}$ ». Leur vie est marquée par la mise en œuvre quotidienne de tactiques et par une position fondamentale de dépendance à l'égard des aides qu'ils reçoivent. Ils sont contraints à des narrations d'euxmêmes constamment diversifiées auprès des donateurs, dontils jouentavecles politiques d'identification et les attentes. D'après deux assistantes sociales travaillant dans une ONG : «Certains prennent les aides et lesvendent... Ily a des gens qui en ont besoin, et d'autres qui les vendent! Parfois ils le font pour acheter des médicaments, mais parfois seulement pour l'argent ! »; « Tu ne peux prendre ton droit, et le droit de l'autre aussi... Voilà, ici ćest la limite de ton droit ${ }^{27}$. "

La figure du réfugié comme menteur est, en quelque sorte, cohérente avec la seule légitimation possible de sa présence, celle de victime ayant besoin d'aide. Quand le réfugié cesse d'habiter le seul lieu légitime, celui du droit à la survie qui lui est accordé par les organisations humanitaires, le corps du réfugié, opaque, devient suspect, et cible de procédures de contrôle ou de jugements moraux. À propos de l'aveu de la part du criminel algérien - considéré mensonger par essence de la part des pouvoirs psychiatrique et juridique français - Frantz Fanon et Raymond Lacaton écrivent: «(...) la réinsertion du criminel par l'aveu de son acte dépend d'une reconnaissance du groupe par l'individu. II ne peut, en somme, y avoir réinsertion s'il n'y avait pas insertion. (...) Ce refus de l'inculpé musulman d'authentifier par l'aveu de son acte le contrat social qu'on lui propose signifie que sa soumission souvent profonde, que nous avons notée en face du pouvoir (judiciaire en l'occurrence), ne peut être confondue avec une acceptation de ce pouvoir ${ }^{28}$. " 
Si l'on n'est pas face à un pouvoir colonial comme celui décrit par Fanon, ces mots font tout de même écho à la figure décrite du Syrien-menteur. Le mensonge peut être appréhendé ici comme une réponse, une résistance face à un univers de procédures arbitraires et impénétrables, dissimulées sous des raisons humanitaires, et dont la légitimité pour le réfugié demeure très problématique. Dans ce territoire donc, le pacte social - et il en est de même pour la relation thérapeutique - constitue une réalité structurellement incertaine, voire inexistante.

Plus que de nous révéler une
La figure du réfugié comme menteur est, en quelque

sorte, cohérente avec la seule

légitimation possible de sa présence, celle de victime

ayant besoin d'aide. caractéristique personnelle, ou d'un groupe, le mensonge nous révèle un champ de relation de pouvoir, l'espace humanitaire, dans lequel le réfugié est inséré, et qui va bien au-delà de la session de psychothérapie ou de l'intervention psychiatrique. Parallèlement, comme le dit Roberto Beneduce : «Ces narrations (...) sont elles-mêmes un exemple contradictoire d'appropriation de l'idéologie humanitaire ${ }^{29}$."

\section{Conclusion}

À travers la définition du Syrien comme un être en manque, ignorant, et, dans certains cas, la référence à la dimension génétique comme cause de la pathologie, les professionnels produisent un discours sur le réfugié qui rabat celui-ci aux marges de l'histoire et du politique.

Ces réflexions nous renvoient à une question centrale, qui apparaît en filigrane : qu'est-ce que « soigner » veut dire, dans ces situations ? La réponse donnée par les professionnels, psychologues et psychiatres est celle qui consiste à renforcer sa capacité d'adaptation à des conditions de vie difficiles, à redevenir fonctionnel ; sa capacité à «faire avec » la situation dans laquelle il vit. Mais qu'est-ce que "s'adapter à ce contexte» veut dire, quand ce contexte même contraint la personne à une survie perpétuelle, dont la seule issue paraît être le voyage dans un autre pays?

L'acte thérapeutique, comme nous l'avons analysé ici, risque de produire donc un dédoublement: à coté de l'exclusion politique, due à la position que le Syrien occupe en tant que réfugié, prennent corps une exclusion et une délégitimation du savoir et de l'expérience du patient, qui empêchent de " penser leurs souffrances et leurs doutes comme un reflet d'une histoire incorporée ${ }^{30} "$.

Le réfugié habite l'espace humanitaire, un espace de tension $^{31}$, dans lequel il doit se soumettre au régime humanitaire qui le veut victime. Tout comme il doit se soumettre à ses mécanismes d'identification et à l'arbitraire de ses procédures bureaucratiques qui le rendent suspect et qui le forcent à jouer de son identité. «Parce qu’il est une négation systématisée de l'autre, une décision forcenée de refuser à l'autre tout attribut d'humanité, le colonialisme accule le peuple dominé à se poser constamment la question: "Qui suis-je en réalitéze ?" ». Pour paraphraser Fanon, on peut se demander : si la maladie est une critique de l'ordre social ${ }^{33}$, le refuge et les dispositifs humanitaires (UNCHR, etc.) ne constituent-ils pas aussi une source de production de la maladie et de la souffrance, dans la mesure où ils participent à l'instauration de ce «régime du quotidien »?

Si les sites humanitaires ne peuvent donc être que des espaces en tension, c'est dans ces espaces en tension que les professionnels de la santé devraient agir, être capables de se situer, sans déhistoriciser l'autre et son propre savoir, en essayant de dépasser les logiques que nous avons tenté de décrire. 